OPEN ACCESS

Edited by:

Giovanni Li Volti,

University of Catania, Italy

Reviewed by:

Susanna lossa

University of Naples Federico II, Italy Gautham Yepuri,

University of Fribourg, Switzerland

*Correspondence:

Jason R. Treberg

jason.treberg@umanitoba.ca

Specialty section

This article was submitted to Integrative Physiology, a section of the journal

Frontiers in Physiology

Received: 31 May 2017

Accepted: 31 August 2017

Published: 15 September 2017

Citation:

Wiens L, Banh S, Sotiri E, Jastroch M, Block BA, Brand MD and Treberg JR (2017) Comparison of Mitochondrial Reactive Oxygen Species Production of Ectothermic and Endothermic Fish

Muscle. Front. Physiol. 8:704

doi: 10.3389/fphys.2017.00704

\section{Comparison of Mitochondrial Reactive Oxygen Species Production of Ectothermic and Endothermic Fish Muscle}

\author{
Lilian Wiens ${ }^{1}$, Sheena Banh ${ }^{1}$, Emianka Sotiri ${ }^{1}$, Martin Jastroch ${ }^{2}$, Barbara A. Block ${ }^{3}$, \\ Martin D. Brand ${ }^{4}$ and Jason R. Treberg ${ }^{1,5 *}$ \\ ${ }^{1}$ Department of Biological Sciences, University of Manitoba, Winnipeg, MB, Canada, ${ }^{2}$ Helmholtz Diabetes Center at \\ Helmholtz Zentrum München, Institute for Diabetes and Obesity, Munich, Germany, ${ }^{3}$ Tuna Research and Conservation \\ Center, Hopkins Marine Station, Stanford University, Stanford, CA, United States, ${ }^{4}$ Buck Institute for Research on Aging, \\ Novato, CA, United States, ${ }^{5}$ Department of Human Nutritional Sciences, University of Manitoba, Winnipeg, MB, Canada
}

Recently we demonstrated that the capacity of isolated muscle mitochondria to produce reactive oxygen species, measured as $\mathrm{H}_{2} \mathrm{O}_{2}$ efflux, is temperature-sensitive in isolated muscle mitochondria of ectothermic fish and the rat, a representative endothermic mammal. However, at physiological temperatures $\left(15^{\circ}\right.$ and $37^{\circ} \mathrm{C}$ for the fish and rat, respectively), the fraction of total mitochondrial electron flux that generated $\mathrm{H}_{2} \mathrm{O}_{2}$, the fractional electron leak (FEL), was far lower in the rat than in fish. Those results suggested that the elevated body temperatures associated with endothermy may lead to a compensatory decrease in mitochondrial ROS production relative to respiratory capacity. To test this hypothesis we compare slow twitch (red) muscle mitochondria from the endothermic Pacific bluefin tuna (Thunnus orientalis) with mitochondria from three ectothermic fishes [rainbow trout (Oncorhynchus mykiss), common carp (Cyprinus carpio), and the lake sturgeon (Acipenser fulvescens)] and the rat. At a common assay temperature $\left(25^{\circ} \mathrm{C}\right)$ rates of mitochondrial respiration and $\mathrm{H}_{2} \mathrm{O}_{2}$ efflux were similar in tuna and the other fishes. The thermal sensitivity of fish mitochondria was similar irrespective of ectothermy or endothermy. Comparing tuna to the rat at a common temperature, respiration rates were similar, or lower depending on mitochondrial substrates. FEL was not different across fish species at a common assay temperature $\left(25^{\circ} \mathrm{C}\right)$ but was markedly higher in fishes than in rat. Overall, endothermy and warming of Pacific Bluefin tuna red muscle may increase the potential for ROS production by muscle mitochondria but the evolution of endothermy in this species is not necessarily associated with a compensatory reduction of ROS production relative to the respiratory capacity of mitochondria.

Keywords: endothermy, ectothermy, tuna, mitochondrial energetics, superoxide, hydrogen peroxide, fractional electron leak 


\section{INTRODUCTION}

Fishes of the family Scombridae (the mackerels, bonitos, and tunas) are highly active marine predators. Within this clade, endothermy has evolved and is most expressed in the three species of bluefin tunas that occupy cooler temperate waters and, as adults, subpolar seas (Block et al., 2005; Boustany et al., 2007; Whitlock et al., 2015). Endothermy is also found in members of the tribe Thunnini (the tunas), and other fishes including cranial endothermy in billfishes and butterfly mackerel, endothermy in lamnid sharks, and systemic endothermy in the Opah (Wegner et al., 2015). Since temperature can affect the rate of biological processes, including enzymatic reaction rates, such endothermy has been a particular focus for comparative physiologists. The research on heat production, evolutionary convergence, and potential selective advantages of endothermy in fishes have been discussed and reviewed elsewhere (for examples see Block, 1994; Block and Finnerty, 1994; Bernal et al., 2001; Dickson and Graham, 2004; Graham and Dickson, 2004).

Bluefin tunas maintain elevated slow-twitch red muscle temperatures in the core of the body, up to $21^{\circ} \mathrm{C}$ above surrounding ambient water temperatures (Stevens et al., 2000; Marcinek et al., 2001; Blank et al., 2007), and swim constantly to respire. Mitochondria must play a key role in resupplying the ATP that is constantly being consumed by muscle contraction. Although, mitochondria are the primary consumers of oxygen during aerobic metabolism they can also produce reactive oxygen species (ROS), primarily as superoxide and $\mathrm{H}_{2} \mathrm{O}_{2}$. ROS production is generally measured as $\mathrm{H}_{2} \mathrm{O}_{2}$ efflux, which combines the net production of superoxide and $\mathrm{H}_{2} \mathrm{O}_{2}$. Excessive production of ROS may lead to macromolecular damage (Murphy, 2009; Jastroch et al., 2010). Studies on mitochondria isolated from ectotherms indicate that the rate of ROS production increases with temperature (Abele et al., 2002; Heise et al., 2003; Chung and Schulte, 2015; Banh et al., 2016); raising the possibility that the warming of specific regions in endothermic fish may also result in increased potential for mitochondrial $\mathrm{H}_{2} \mathrm{O}_{2}$ efflux. If higher physiological temperatures could lead to elevated potential for ROS production then compensatory mechanisms to minimize mitochondrial $\mathrm{H}_{2} \mathrm{O}_{2}$ production may co-evolve with endothermy.

We recently demonstrated that $\mathrm{H}_{2} \mathrm{O}_{2}$ efflux from muscle mitochondria isolated from three ectothermic fishes was much lower than rates for rat muscle mitochondria when assayed at physiological temperatures of $15^{\circ}$ and $37^{\circ} \mathrm{C}$ for the ectothermic fish and rat respectively (Banh et al., 2016). However, the fractional electron leak (FEL), a value that normalizes ROS production relative to total mitochondrial electron flux, was markedly higher for fish mitochondria compared to the rat at physiological temperatures (Banh et al., 2016). The high FEL in the fish suggests that relative to the overall metabolic capacity of the mitochondria (including the electron transport chain and Krebs cycle where the majority of superoxide $/ \mathrm{H}_{2} \mathrm{O}_{2}$ producing complexes are found) ectothermic fish mitochondria have intrinsically higher potential for $\mathrm{H}_{2} \mathrm{O}_{2}$ production compared to the rat. For all species examined the rate of mitochondrial $\mathrm{H}_{2} \mathrm{O}_{2}$ production was greater at higher temperatures. Indeed, in some cases, mitochondria from trout and carp produced as much or more $\mathrm{H}_{2} \mathrm{O}_{2}$ at $25^{\circ} \mathrm{C}$ than rat mitochondria did at $37^{\circ} \mathrm{C}$ (Banh et al., 2016). Taken together these findings suggest that muscle mitochondria from ectotherms may have a greater propensity for $\mathrm{H}_{2} \mathrm{O}_{2}$ production compared to a representative endotherm.

Our recent work proposed that mitochondrial $\mathrm{H}_{2} \mathrm{O}_{2}$ producing and consuming pathways have differing temperature sensitivities (Banh et al., 2016). This thermal mismatch hypothesis of mitochondrial $\mathrm{H}_{2} \mathrm{O}_{2}$ metabolism (Banh et al., 2016) predicts that compensatory mechanisms may have been crucial in the evolution of endothermy because excess ROS production would be associated with oxidative stress and disruption of redox homeostasis. Thus, one can hypothesize that some compensatory response to mitigate the influence of elevated body temperatures could be concomitant with the evolution of endothermy. The current study tests this hypothesis by comparing mitochondria isolated from red muscle of the endothermic Pacific Bluefin tuna (Thunnus orientalis), maintained at an elevated $25^{\circ} \mathrm{C}$, with three ectothermic fishes at $15^{\circ} \mathrm{C}$. For reference we also evaluate the well-studied rat as a representative endothermic mammal. For all species the respiratory capacity and rate of $\mathrm{H}_{2} \mathrm{O}_{2}$ formation was measured at the respective physiological temperatures and a common temperature of $25^{\circ} \mathrm{C}$ to test if tuna had reduced potential for ROS formation relative to their mitochondrial metabolic capacity (estimated by respiration rate). Overall, we find that tuna red muscle mitochondria are similar to those of ectothermic fish species in both the capacity and thermal sensitivity of ROS formation. Thus, enhanced mitochondrial superoxide $/ \mathrm{H}_{2} \mathrm{O}_{2}$ production may be an underappreciated consequence of the tissue warming found in the muscle of endothermic fishes.

\section{MATERIALS AND METHODS}

\section{Animals and Sampling}

All animal use procedures were based on the policies from the Canadian Council on Animal Care and approved by the University of Manitoba Fort Garry Campus Animal Care Committee (for ectothermic fish and rat) or the Stanford Administrative Panel on Laboratory Animal Care Committee in accordance to all policies of Stanford University (tuna).

\section{Pacific Bluefin Tuna}

Pacific bluefin tuna ( $T$. orientalis) were housed at the Tuna Research and Conservation Center at Stanford University's Hopkins Marine Station, Pacific Grove, CA. Animal collection, transport and husbandry were the same as previously reported (Galli et al., 2009; Clark et al., 2010) and all animals in this study were acclimated to $20^{\circ} \mathrm{C}$ prior to experimental initiation for at least 30 days. Fish were of both sexes and $\sim 13-15$ $\mathrm{kg}$ and $80-90 \mathrm{~cm}$ in fork length. For sampling, fish were killed by pithing, followed by spinal severance at the junction between the spine and skull. Tissues were immediately removed and placed in ice-cold isolation medium for preparation of mitochondria. 


\section{Ectothermic Fish}

The ectothermic fishes in this study include two other teleosts, the relatively high aerobic capacity rainbow trout (Oncorhynchus mykiss) and the low activity benthic-dwelling common carp (Cyprinus carpio) as well as the lake sturgeon (Acipenser fulvescens), a relatively inactive Acipenseriform species. Rainbow trout and carp were obtained from commercial suppliers. Lake sturgeon were reared from eggs fertilized at the University of Manitoba. All ectothermic fish were maintained at $15^{\circ} \mathrm{C}$, for at least 8 weeks, on a 12:12 photoperiod in flow-through tanks and fed commercial pelleted food to satiation at least every other day. Fish were of both sexes and were $\sim 1.5-4.5 \mathrm{~kg}$. For sampling, fish were killed by a blow to the head, followed by severing of the gill arches, and tissues were immediately dissected out and placed in ice-cold isolation medium.

\section{Rats}

Male Sprague-Dawley rats, $\sim 250-400$ g, were from the University of Manitoba and housed at the Duff-Roblin Animal Holding facility in standard cages with wood shaving or cardboard bedding along with shelters, washers and other plastic environmental enrichment. Rats had ad libitum access to chow and water. Rats were killed by asphyxiation with $\mathrm{CO}_{2}$ followed by pneumothorax and hindlimb muscle was dissected off and put in ice-cold isolation medium prior to processing for isolation of mitochondria.

\section{Mitochondrial Isolation}

Tissues were rinsed in ice-cold isolation medium and diced to facilitate homogenization. Two isolation media were used in this study. For tuna, we used $140 \mathrm{mM} \mathrm{KCl,} 20 \mathrm{mM}$ HEPES, $2 \mathrm{mM}$ EGTA, pH 7.0 at $20^{\circ} \mathrm{C}$ whereas $120 \mathrm{mM} \mathrm{KCl,} 20 \mathrm{mM}$ HEPES, $1 \mathrm{mM}$ EGTA, pH 7.1 at $20^{\circ} \mathrm{C}$ was used for the rats and carp. For rainbow trout and sturgeon we tested both isolation media and found no difference in results. All steps were maintained at $0-4^{\circ} \mathrm{C}$. The procedure for rats (Affourtit et al., 2012) and the ectothermic fishes (Banh et al., 2016) are described in detail elsewhere but, briefly, involved incubating diced tissue with a protease followed by disruption by homogenization and isolation of mitochondria by differential centrifugation. The final pellet was resuspended in a small volume of isolation medium at $\sim 20$ $45 \mathrm{mg}$ mitochondrial protein $\cdot \mathrm{ml}^{-1}$ (determined by biuret assay with BSA as standard).

For tuna, diced tissue was mixed with ice-cold homogenization medium (isolation medium plus $0.5 \%$ w/v BSA) and homogenized with a motor-driven Teflon (Polytetrafluoroethylene) to glass homogenizer. The homogenate was centrifuged for $5 \mathrm{~min}$ at $900 \times g$. The supernatant was collected and filtered through a fine plastic mesh to aid in lipid and particulate removal. Following filtration, the supernatant was centrifuged at $9,000 \times g$ for $10 \mathrm{~min}$. The resulting pellet was washed by resuspending in isolation medium followed by centrifugation $9,000 \times g$ for $10 \mathrm{~min}$. Care was taken to not transfer any of the lipid in the supernatant or resuspend any material that was clearly not mitochondria (for example red blood cells and particulate matter). The resulting pellet was washed a second time (as described above) and the final pellet was resuspended in a small volume of isolation medium at
30-60 mg mitochondrial protein $\cdot \mathrm{ml}^{-1}$ (determined by biuret assay with BSA as standard).

\section{Mitochondrial $\mathrm{H}_{2} \mathrm{O}_{2}$ Production}

Combined superoxide and hydrogen peroxide production by mitochondria was measured spectrofluorometrically as $\mathrm{H}_{2} \mathrm{O}_{2}$ efflux in the same medium as used for respiration. In some cases phosphate was omitted or $1 \mu \mathrm{g} \cdot \mathrm{ml}^{-1}$ oligomycin was added, we found these had relatively little (c. $10 \%$ or less) influence on the observed rates and thus pooled all data. The assay medium also contained an $\mathrm{H}_{2} \mathrm{O}_{2}$ detection system consisting of $5 \mathrm{IU} \cdot \mathrm{ml}^{-1}$ of horseradish peroxidase, $25 \mathrm{IU} \cdot \mathrm{ml}^{-1}$ superoxide dismutase, and $50 \mu \mathrm{M}$ Amplex UltraRed in a total volume of $2 \mathrm{ml}$ using either an Agilent Eclipse or a Shimadzu RF-5301PC. Fluorescence was monitored, with constant stirring, at $560 \mathrm{~nm}$ excitation and $590 \mathrm{~nm}$ emission wavelengths, respectively. Raw fluorescence values were transformed to moles of $\mathrm{H}_{2} \mathrm{O}_{2}$ based on calibration with known amounts of $\mathrm{H}_{2} \mathrm{O}_{2}$. Background rates of product formation prior to the addition of substrate were low (typically $<10 \%$ of rate with substrate) and were subtracted from rates in the presence of substrate.

\section{Mitochondrial Respiration}

Mitochondrial substrate oxidation was measured in a waterjacketed chamber with a Rank Brothers Clark-type oxygen electrode (Cambridge, U.K) or using the Oroboros $\mathrm{O} 2 \mathrm{~K}$ (Innsbruck, Austria). Tuna mitochondria used the following respiration medium: $140 \mathrm{mM} \mathrm{KCl,} 20 \mathrm{mM}$ Hepes, $1 \mathrm{mM}$ EGTA, $5 \mathrm{mM} \mathrm{K}_{2} \mathrm{HPO}_{4}$, and $0.5 \%(\mathrm{w} / \mathrm{v}) \mathrm{BSA}, \mathrm{pH} 7.3$ at $20^{\circ} \mathrm{C}$. For ectotherm fish and rats the standard medium was $120 \mathrm{mM} \mathrm{KCl}$, $20 \mathrm{mM}$ Hepes, $1 \mathrm{mM}$ EGTA, $5 \mathrm{mM} \mathrm{K}_{2} \mathrm{HPO}_{4}$, and $0.3 \%(\mathrm{w} / \mathrm{v})$ BSA, pH 7.4 at $20^{\circ} \mathrm{C}$. Note, we found comparable (within 10\%) results between the two respiration media with the ectothermic fish mitochondria.

Following addition of mitochondria to the chamber the rates of oxygen consumption were measured under the following respiratory conditions: (i) non-phosphorylating, in the presence of exogenous substrate (state 2); (ii) phosphorylating, the rate with exogenous substrate plus 500 or $800 \mu \mathrm{M}$ ADP (state 3); (iii) a pharmacologically induced non-phosphorylating state, with 1 $\mu \mathrm{g} \cdot \mathrm{ml}^{-1}$ oligomycin (which blocks ATP synthesis) (state 4o). These conditions were measured in sequence and the respiratory control ratios (RCR) were calculated as the rate of oxygen consumption of mitochondria in state $3 /$ state 4 o.

\section{Data Analysis}

All data are shown as mean \pm SEM. Values were compared either by ANOVA (with Student-Newman-Keuls post-hoc test) or $t$-test (Welch's) with $p<0.05$ being considered significant.

To determine the FEL we calculated values based on:

$$
\begin{aligned}
\text { FEL }= & 100 \times\left[\left(\text { rate of } \mathrm{H}_{2} \mathrm{O}_{2} \text { efflux }\right) \times\left(\text { rate of } \mathrm{H}_{2} \mathrm{O}_{2}\right. \text { efflux }\right. \\
& \left.\left.+ \text { rate of } \mathrm{O}_{2} \text { consumption }\right)^{-1}\right]
\end{aligned}
$$

Where $\mathrm{H}_{2} \mathrm{O}_{2}$ efflux rate is in nmol $\min ^{-1} \mathrm{mg}$ protein ${ }^{-1}$ and

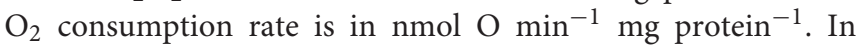
some cases rates of $\mathrm{H}_{2} \mathrm{O}_{2}$ efflux and $\mathrm{O}_{2}$ consumption could 
not be measured at all assay temperatures for mitochondria isolated from the same individual animal therefore values are expressed as mean \pm SEM where the SEM was derived by standard error propagation procedures. Differences among these mean values were compared by 2 -tailed Welch's $t$-test $(p<0.05$ being considered significant).

\section{RESULTS}

\section{Interspecific Patterns in Rates of $\mathrm{H}_{2} \mathrm{O}_{2}$ Production}

Aerobic locomotion in both fish and mammals is largely fuelled by carbohydrate and lipid oxidation (Lauff and Wood, 1996, 1997; McClelland, 2004), as such, we evaluated the potential for mitochondrial ROS formation during the oxidation of either pyruvate or palmitoylcarnitine to reflect these respective fuels. In both cases saturating malate was also included to alleviate the trapping of intramitochondrial coenzyme-A as acetyl-CoA by the provisioning of oxaloacetate and to supply NADH for complex I via malate dehydrogenase. When compared at physiological temperatures, $15^{\circ}, 25^{\circ}$, and $37^{\circ} \mathrm{C}$ for ectothermic fish, Bluefin tuna and rat respectively, there was a pattern of increasing capacity for ROS production as physiological temperature increased but the tuna mitochondrial ROS production at $25^{\circ} \mathrm{C}$ was comparable to the rat mitochondria at $37^{\circ} \mathrm{C}$ (Figure 1).

In all species the mitochondrial $\mathrm{H}_{2} \mathrm{O}_{2}$ production was sensitive to temperature (Figure 1) with similar sensitivity across species. At higher temperatures the rate of $\mathrm{H}_{2} \mathrm{O}_{2}$ production was increased. Overall the rates of $\mathrm{H}_{2} \mathrm{O}_{2}$ production by isolated Pacific bluefin tuna mitochondria were comparable to other fishes. When measured at a common assay temperature of $25^{\circ} \mathrm{C}$, the physiological temperature for the bluefin tuna red muscle, rates of $\mathrm{H}_{2} \mathrm{O}_{2}$ production by tuna mitochondria were not different from mitochondria isolated from the other fishes (Figure 1). In all cases the rates at $25^{\circ} \mathrm{C}$ in fish mitochondria were markedly higher than the rate seen with rat muscle mitochondria with both carbohydrate and lipid based oxidative substrates (Figure 1). Within species we found no pattern of difference in rates of $\mathrm{H}_{2} \mathrm{O}_{2}$ formation between oxidation of pyruvate or palmitoylcarnitine (Figures 1A,B).

\section{Comparisons of Respiratory Rates}

To evaluate if the differences seen in $\mathrm{H}_{2} \mathrm{O}_{2}$ production rates might be linked to mitochondrial respiratory capacity we measured the rates of respiration at physiological temperatures as well as at a common temperature of $25^{\circ} \mathrm{C}$. Under conditions of phosphorylating respiration (presence of ADP) and nonphosphorylating respiration (absence of ADP phosphorylation) there was an overall tendency for increasing rates with increasing physiological temperature (Figure 2). At the common temperature of $25^{\circ} \mathrm{C}$ the rates of respiration with either pyruvate or palmitoylcarnitine (both supplemented with malate) were not different across fish mitochondria, with the exception of sturgeon being higher than trout with pyruvate and malate under phosphorylating conditions (Figure 2). Rates of respiration were generally higher for rat mitochondria. The respiratory control ratio (RCR) was not affected by assay temperature except for trout (Figure 2C) and at $25^{\circ} \mathrm{C}$ the RCR for Pacific bluefin tuna was not different from the other fishes. The stability of RCR values across temperatures suggests collapsing mitochondrial coupling efficiency with changing temperature is an unlikely explanation for differences in $\mathrm{H}_{2} \mathrm{O}_{2}$ production with assay temperature.

\section{Fractional Electron Leak}

At a common assay temperature of $25^{\circ} \mathrm{C}$ the $\mathrm{FEL}$ for all fish species was markedly greater than for rat, with Pacific
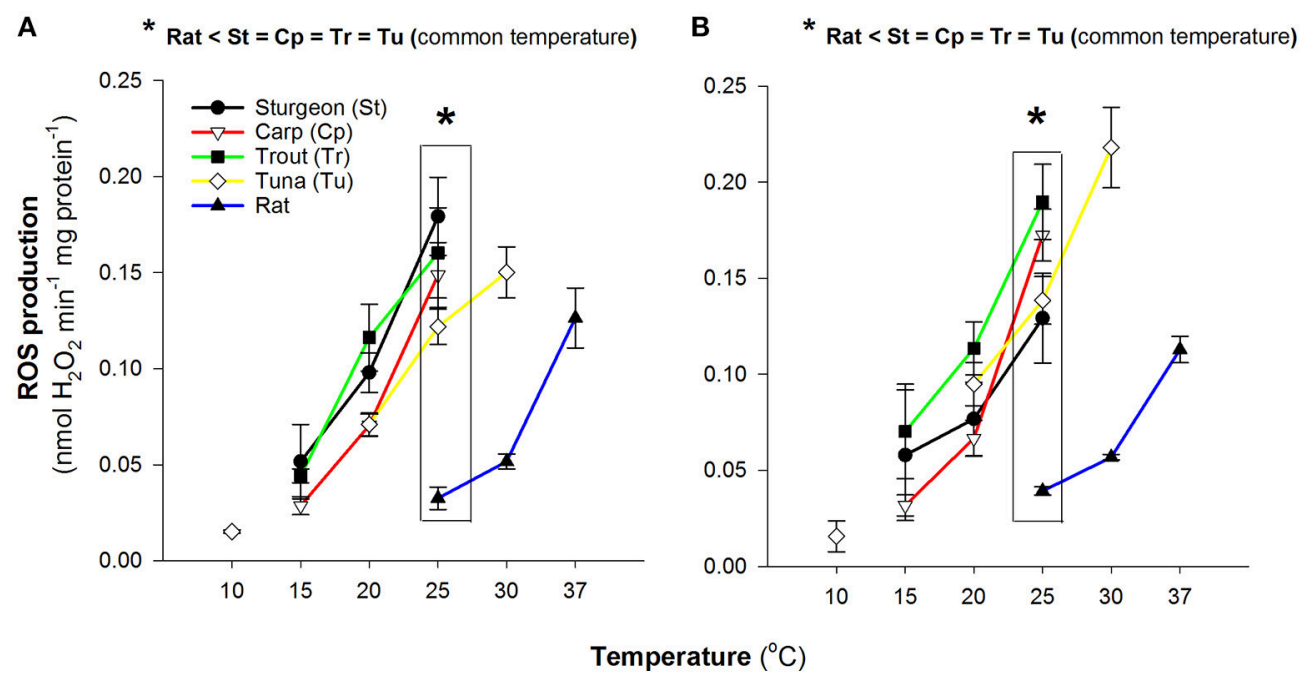

FIGURE 1 | Comparison of reactive oxygen species production by isolated muscle mitochondria. Data are mean \pm SEM ( $n=3-5$ depending on species) and substrates were $5 \mathrm{mM}$ pyruvate and malate (A) or $0.05 \mathrm{mM}$ D/L-palmitoylcarnitine and $5 \mathrm{mM}$ malate (B). Assay temperature is indicated and rates were determined as $\mathrm{H}_{2} \mathrm{O}_{2}$ efflux normalized to mitochondrial protein. Physiologically relevant temperatures are $15^{\circ} \mathrm{C}$ for sturgeon, carp and trout; $25^{\circ} \mathrm{C}$ for $\mathrm{Bluefin}$ tuna; $37^{\circ} \mathrm{C}$ for rat. 

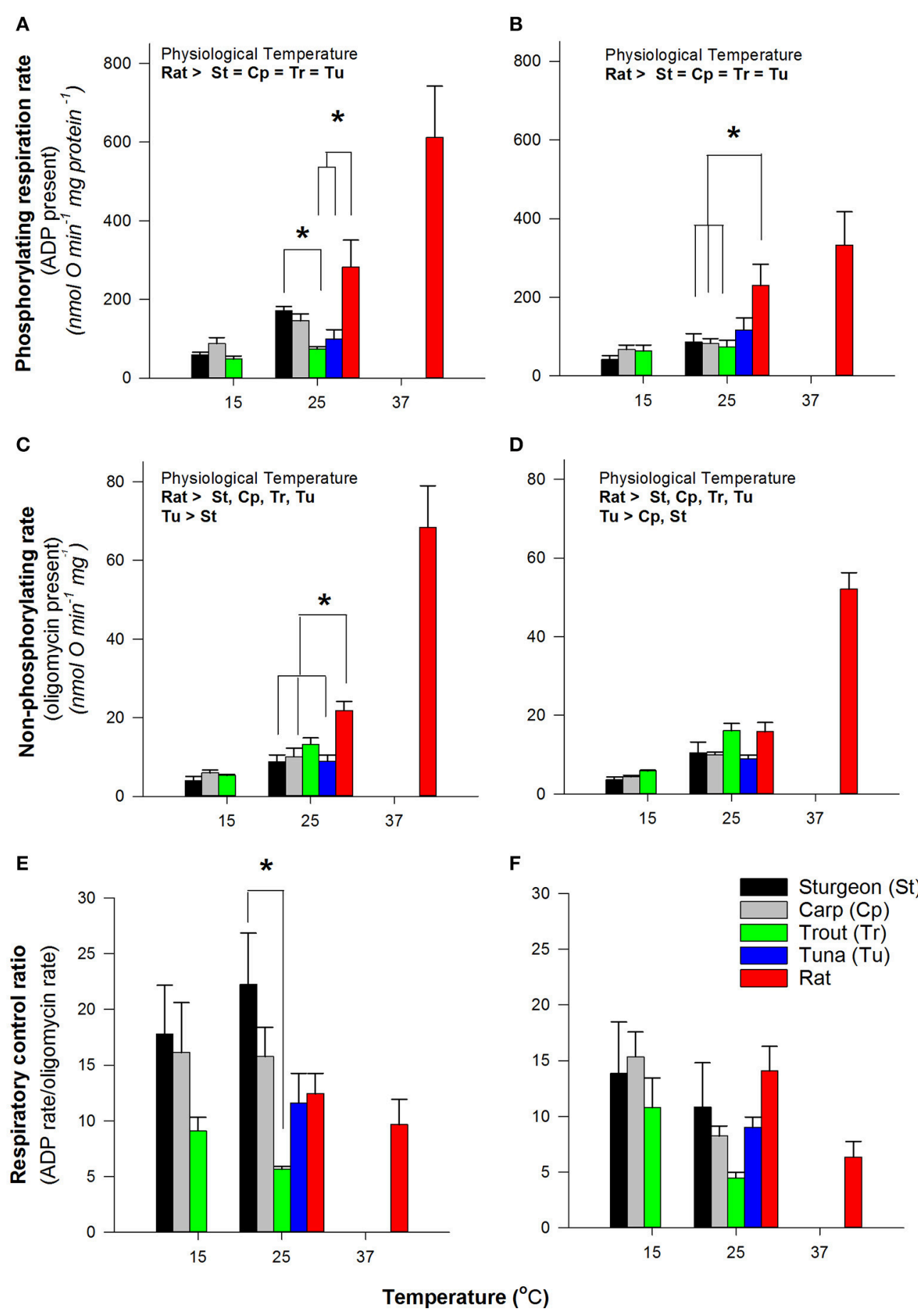

FIGURE 2 | Comparison of respiration rates by isolated muscle mitochondria. Data are mean \pm SEM ( $n=3-5$ depending on species) and substrates were 5 mM pyruvate and malate $(\mathbf{A}, \mathbf{C}, \mathbf{E})$ or $0.05 \mathrm{mM}$ D/L-palmitoylcarnitine and $5 \mathrm{mM}$ malate (B,D,F). Assay temperature is indicated and contrasts were made at a common temperature (indicated by * and brackets) or physiological temperatures as indicated within figures. Physiologically relevent temperatures are $15^{\circ} \mathrm{C}$ for sturgeon, carp and trout; $25^{\circ} \mathrm{C}$ for Bluefin tuna; $37^{\circ} \mathrm{C}$ for rat.

bluefin tuna being unexceptional in comparison to the other fish (Figure 3). For species where two test temperatures were used (all species but the tuna) there was a tendency for increasing FEL with higher assay temperature. This thermal sensitivity led to some ectothermic fishes having values for FEL that were not different from the rat at physiological temperatures (Figure 3), but the high levels of variation warrant some caution with interpretation of this result. 


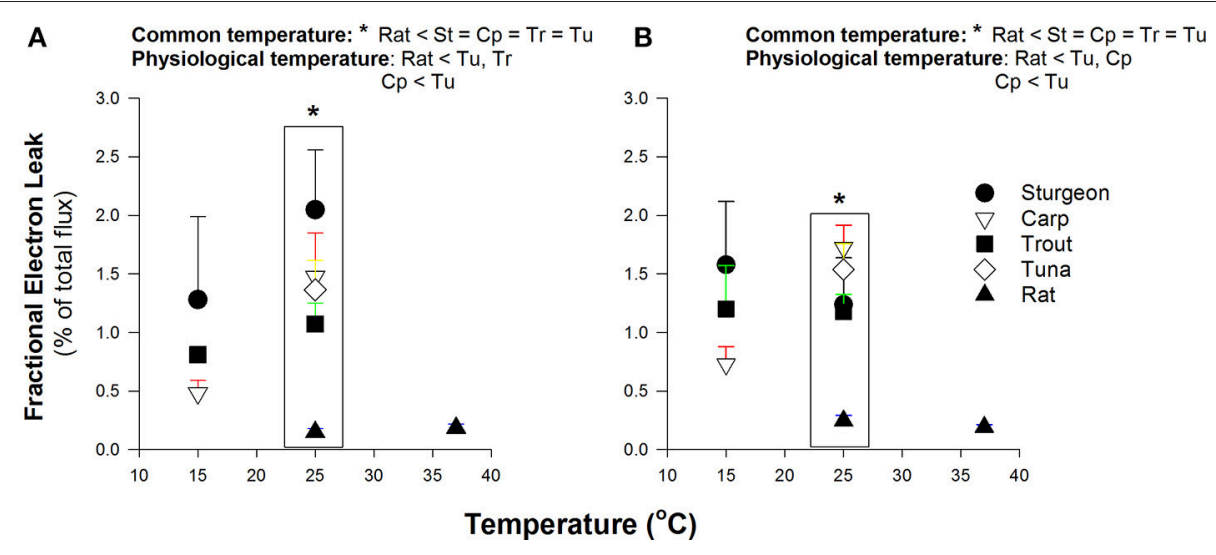

FIGURE 3 | Fractional electron leak by isolated muscle mitochondria. Data are mean \pm SEM ( $n=3-5$ depending on species) and substrates were 5 mM pyruvate and malate (A) or $0.05 \mathrm{mM} \mathrm{D/L-palmitoylcarnitine} \mathrm{and} 5 \mathrm{mM}$ malate (B). Assay temperature is indicated and contrasts were made at a common temperature (indicated by * and brackets) or physiological temperatures as indicated within figures. Physiologically relevent temperatures are $15^{\circ} \mathrm{C}$ for sturgeon, carp and trout; $25^{\circ} \mathrm{C}$ for Pacific bluefin tuna; $37^{\circ} \mathrm{C}$ for rat. Species abbreviations are as indicated in Figures 1, 2.

\section{DISCUSSION}

\section{Comparisons among Fishes}

For all species examined the production of $\mathrm{H}_{2} \mathrm{O}_{2}$ by isolated muscle mitochondria was a function of assay temperature. Higher assay temperatures led to higher rates of production (Figure 1). However, across assay temperatures and using all substrate mixes tested, the rates of $\mathrm{H}_{2} \mathrm{O}_{2}$ production by isolated Pacific bluefin tuna mitochondria compared favorably with other fish mitochondria. Similarly, Pacific bluefin tuna mitochondria had similar respiration rates to most other fish red muscle mitochondria when measured at a common assay temperature irrespective of the physiological body temperature for the mitochondria (Figure 2). Our respiration rates with pyruvate also compare well with those for other teleosts over a range of both in vitro assay and physiological temperatures (Johnston et al., 1994, 1998) suggesting our mitochondrial preparations are reasonably consistent with those used in other studies on temperature effects on fish mitochondria.

A potential way to separate mitochondrial $\mathrm{H}_{2} \mathrm{O}_{2}$ production from the mitochondrial roles in oxygen consumption is to express the electron leakage to $\mathrm{H}_{2} \mathrm{O}_{2}$ as a fraction of the overall electron flux (FEL). Using the FEL we find no evidence for reduced potential for $\mathrm{H}_{2} \mathrm{O}_{2}$ formation relative to substrate oxidation rates; tuna mitochondria were similar to mitochondria from ectothermic fishes (Figure 3). The respiratory capacity, measured as ADP-stimulated respiration rate, was similar in Pacific bluefin tuna mitochondria to most other fish red muscle mitochondria. This similarity in respiration rate is consistent with no compensatory change in the endothermic tuna red muscle in the overall metabolic systems responsible for electron flux to oxygen either as respiration or leaking as mitochondrial $\mathrm{H}_{2} \mathrm{O}_{2}$ production. Of note, one could argue that the ADPstimulated rate of oxygen consumption better reflects the overall amount of biochemical "machinery" in the mitochondrion, and thus the phosphorylating rate of respiration (state 3 ) is a better denominator to normalize $\mathrm{H}_{2} \mathrm{O}_{2}$ production; however, we find the same overall pattern is seen as that for FEL indicating that regardless of the respiration rate used to normalize $\mathrm{H}_{2} \mathrm{O}_{2}$ production all fish muscle mitochondria appear comparable at the same assay temperature (data not shown).

\section{Comparison of Muscle Mitochondria from Fish and the Rat}

We included one representative mammal, the rat. Overall, mitochondria from all fish assayed appear to have greater potential for electron leak relative to their respiratory capacity (FEL) compared to the rat. The similarity of FEL across fishes in this study suggests fishes, and possibly other ectotherms, may have a propensity toward increased electron leakage compared to more derived endothermic species. This may represent an adaption to minimize the ROS induced damage that could occur at higher endogenous temperatures common in mammals and birds. This pattern warrants further investigation to determine if it holds more broadly across taxa. Contrasting endothermic and ectothermic representative birds and reptiles would be a particularly interesting comparison along these lines.

\section{Fuel Selection: Is There a Trade-Off between $\mathrm{H}_{2} \mathrm{O}_{2}$ Production and Specific Carbon Source?}

During aerobic locomotion both lipid and carbohydrate are important carbon sources for oxidation in teleost fishes (Lauff and Wood, 1996, 1997) and mammals (McClelland, 2004). This is consistent with relatively similar rates of pyruvate and fatty acid oxidation by isolated muscle mitochondria from teleosts as seen by us and others (Moyes et al., 1989, 1992; Chamberlin et al., 1991). Thus, pyruvate and palmitoylcarnitine were used as representative carbohydrate- and lipid-based oxidative substrates in the present study.

These physiologically important substrates were also chosen because they are known to produce $\mathrm{H}_{2} \mathrm{O}_{2}$ in isolated mitochondria (reviewed in Brand, 2010). Pyruvate and 
palmitoylcarnitine may contribute directly to mitochondrial ROS production via the matrix enzymes involved with their metabolism, as well as indirectly by producing $\mathrm{NADH}$ and $\mathrm{QH}_{2}$ which reduce electron transport chain complexes I and III respectively. It has been reported that palmitoylcarnitine driven respiration in rodent mitochondria may have high capacity for $\mathrm{H}_{2} \mathrm{O}_{2}$ production compared to other NADH-generating substrates (St Pierre et al., 2002; Seifert et al., 2010); although more recent work found the rates comparable to that found with other NADH-generating substrates (Perevoshchikova et al., 2013). If lipid fuelled respiration was to lead to greater potential for $\mathrm{H}_{2} \mathrm{O}_{2}$ production then there is a possibility that the enhanced lipid oxidation proposed for high-performance fishes (Weber and Haman, 1996) may have consequences on mitochondrial $\mathrm{H}_{2} \mathrm{O}_{2}$ production. However, we broadly find comparable rates of $\mathrm{H}_{2} \mathrm{O}_{2}$ production, FEL, and ADP-stimulated respiration rates with isolated muscle mitochondria from several fishes and one mammal. As such, our evidence does not support a trade-off between selection for lipid or carbohydrate oxidation in muscle and potential for $\mathrm{H}_{2} \mathrm{O}_{2}$ production.

\section{Limitations of the Study ADP Availability in Fish Red Muscle}

The rate of $\mathrm{H}_{2} \mathrm{O}_{2}$ production from mitochondria isolated from several tissues, including brain, liver and muscle, typically declines in the presence of ADP (Starkov and Fiskum, 2003; Starkov et al., 2004; Santiago et al., 2008; Quinlan et al., 2012; Goncalves et al., 2015). This decline appears to be primarily due to decreased protonmotive force and the consequent decreased reduction state of electron carriers and the enzyme complexes involved with electron transport and substrate oxidation. Although, some ADP is always being produced in skeletal muscle due to ATP hydrolysis, we are unaware of precise data to estimate the exact ADP availability typical in the red muscle of the various fish species we examined here. For this reason we use the rate of $\mathrm{H}_{2} \mathrm{O}_{2}$ production in the absence of ADP as a worst case scenario of ROS production under conditions of saturating substrate and oxygen availability. Even though the ADP availability in red muscle of constantly swimming fish should alleviate some ROS formation, our results suggest that mitochondria in a teleost with elevated muscle temperature should be exposed to a greater $\mathrm{H}_{2} \mathrm{O}_{2}$ production rate than if the same mitochondria were respiring at a lower temperature.

\section{Underestimations of $\mathrm{H}_{2} \mathrm{O}_{2}$ Production Due to Matrix Consumers}

Using $\mathrm{H}_{2} \mathrm{O}_{2}$ efflux as a measure of $\mathrm{H}_{2} \mathrm{O}_{2}$ production by isolated mitochondria underestimates the actual production and with rat muscle mitochondria this underestimate is a function of the production rate, with high rates leading to lower underestimates (Munro et al., 2016). It was not possible to undertake the complex procedure to compromise the matrix $\mathrm{H}_{2} \mathrm{O}_{2}$ consuming pathways (see Munro et al., 2016 for full details) and thus we cannot exclude the possibility that patterns seen here may not fully reflect the actual rates of production for each species. As such, it is possible a compensatory increase in antioxidant capacity is found in Bluefin tuna that our dataset may not capture. Alternatively, the increased antioxidant capacity with increasing temperature, even if incomplete as proposed elsewhere (Banh et al., 2016), may be sufficient to compensate for increase $\mathrm{H}_{2} \mathrm{O}_{2}$ production capacity.

\section{Size Scaling Effects}

The production of $\mathrm{H}_{2} \mathrm{O}_{2}$ by isolated mitochondria may scale with body size with larger animals having lower rates of $\mathrm{H}_{2} \mathrm{O}_{2}$ production than smaller species (Lambert et al., 2007). We have not tried to account for mass-scaling effects but in the case of the rat, the smallest species used, it would be expected this pattern would disproportionately elevate $\mathrm{H}_{2} \mathrm{O}_{2}$ production. Conversely, rat has the lowest rate of $\mathrm{H}_{2} \mathrm{O}_{2}$ formation at a common assay temperature. For the fishes, the Pacific bluefin tuna was the largest of the species and thus may have somewhat lower rates of $\mathrm{H}_{2} \mathrm{O}_{2}$ production due to scaling effects. Even if the bluefin tuna $\mathrm{H}_{2} \mathrm{O}_{2}$ values are low due to their larger size this would not change our primary thesis in this study: there is no support for reduced $\mathrm{H}_{2} \mathrm{O}_{2}$ production by isolated muscle mitochondria from this endothermic fish species since correcting for mass would be expected to increase the rates in tuna relative to smaller fish species.

\section{Muscle Fiber Type}

Finally, all fish muscle mitochondrial preparations were from red muscle, which is almost exclusively slow-twitch muscle because of the anatomical separation of slow and fast twitch fibers in most fishes making fish excellent models for studying muscle mitochondrial function from specific fiber types (Leary et al., 2003). However, the rat muscle preparation will be a mix of fiber types and it has been reported that the $\mathrm{H}_{2} \mathrm{O}_{2}$ formation varies by fiber type in rodent muscle (Anderson and Neufer, 2006). But again the pattern found should have biased the rat mitochondrial preparation toward elevated $\mathrm{H}_{2} \mathrm{O}_{2}$ production because fast-twitch muscle appears to produce more $\mathrm{H}_{2} \mathrm{O}_{2}$ than slow-twitch fibers (Anderson and Neufer, 2006). Therefore, we conclude that our finding of markedly lower $\mathrm{H}_{2} \mathrm{O}_{2}$ formation in the representative mammal should not be compromised but the use of a mixed muscle type for this species alone.

\section{Summary}

The elevated red muscle temperature in endothermic fishes should increase the potential for $\mathrm{H}_{2} \mathrm{O}_{2}$ formation but we see no evidence for compensatory decrease in $\mathrm{H}_{2} \mathrm{O}_{2}$ production in the Pacific Bluefin tuna, whereas we do see this in a representative endothermic mammal. Slow-twitch red muscle has a primary function in locomotion in these fish. This suggests that there is a trade-off in red, slow-twitch muscle between thermal effects that are selected for to improve muscle performance at elevated temperature (Altringham and Block, 1997), and the propensity toward mitochondrial $\mathrm{H}_{2} \mathrm{O}_{2}$ formation in the evolution of endothermy in fishes.

\section{AUTHOR CONTRIBUTIONS}

LW: Helped write initial manuscript draft, contributed original data and data analysis; SB: Contributed original data, analysis and 
editorial contribution to manuscript; ES: Contributed original data and editorial contribution to manuscript; MJ: Contributed to study design, provided original data, and assisted in writing manuscript; BB: Contributed to study design and with writing of manuscript; $\mathrm{MB}$ : Contributed to study design and with writing of manuscript; JT contributed to study design, provided original data, and assisted in writing manuscript.

\section{FUNDING}

Funding was provided by a NSERC Discovery Grant (\#418503 to JT) and the Canada Research Chairs Program (\#223744 to

\section{REFERENCES}

Abele, D., Heise, K., Portner, H. O., and Puntarulo, S. (2002). Temperaturedependence of mitochondrial function and production of reactive oxygen species in the intertidal mud clam Mya arenaria. J. Exp. Biol. 205, 1831-1841.

Affourtit, C., Quinlan, C. L., and Brand, M. D. (2012). Measurement of proton leak and electron leak in isolated mitochondria. Methods Mol. Biol. 810, 165-182. doi: 10.1007/978-1-61779-382-0_11

Altringham, J. D., and Block, B. A. (1997). Why do tuna maintain elevated slow muscle temperatures? Power output of muscle isolated from endothermic and ectothermic fish. J. Exp. Biol. 200, 2617-2627.

Anderson, E. J., and Neufer, P. D. (2006). Type II skeletal myofibers possess unique properties that potentiate mitochondrial $\mathrm{H}_{2} \mathrm{O}_{2}$ generation. Am. J. Physiol. Cell Physiol. 290, C844-C851. doi: 10.1152/ajpcell.00402.2005

Banh, S., Wiens, L., Sotiri, E., and Treberg, J. R. (2016). Mitochondrial reactive oxygen species production by fish muscle mitochondria: potential role in acute heat-induced oxidative stress. Comp. Biochem. Physiol. B 191, 99-107. doi: 10.1016/j.cbpb.2015.10.001

Bernal, D., Dickson, K. A., Shadwick, R. E., and Graham, J. B. (2001). Review: analysis of the evolutionary convergence for high performance swimming in lamnid sharks and tunas. Comp. Biochem. Physiol. A 129, 695-726. doi: 10.1016/S1095-6433(01)00333-6

Blank, J. M., Morrissette, J. M., Farwell, C. J., Price, M., Schallert, R. J., and Block, B. A. (2007). Temperature effects on metabolic rate of juvenile Pacific bluefin tuna Thunnus orientalis. J. Exp. Biol. 210, 4254-4261. doi: 10.1242/jeb. 005835

Block, B. A. (1994). Thermogenesis in muscle. Annu. Rev. Physiol. 56, 535-577. doi: 10.1146/annurev.ph.56.030194.002535

Block, B. A., and Finnerty, J. R. (1994). Endothermy in fishes: a phylogenetic analysis of constraints, predispositions, and selection pressures. Environ. Biol. Fishes 40, 283-302. doi: 10.1007/BF00002518

Block, B. A., Teo, S. L. H., Walli, A., Boustany, A., Stokesbury, M. J. W., Farwell, C. J., et al. (2005). Electronic tagging and population structure of Atlantic bluefin tuna. Nature 434, 1121-1127. doi: 10.1038/nature03463

Boustany, A. M., Matteson, R., Castleton, M., Farwell, C., and Block, B. A. (2007). Movements of Pacific bluefin tuna (Thunnus orientalis) in the Eastern North Pacific revealed with archival tags. Prog. Oceanogr. 86, 94-104. doi: $10.1016 / j$.pocean.2010.04.015

Brand, M. D. (2010). The sites and topology of mitochondrial superoxide production. Exp. Geront. 45, 466-472. doi: 10.1016/j.exger.2010.01.003

Chamberlin, M. E., Glemet, H. C., and Ballantyne, J. S. (1991). Glutamine metabolism in a holostean (Amia calva) and teleost fish (Salvelinus namaycush). Am. J. Physiol. 260, R159-R166.

Chung, D. J., and Schulte, P. M. (2015). Mechanisms and costs of mitochondrial thermal acclimation in a eurythermal killifish (Fundulus heteroclitus). J. Exp. Biol. 218, 1621-1631. doi: 10.1242/jeb.120444

Clark, T. D., Brandt, W. T., Nogueira, J., Rodriguez, L. E., Price, M., Farwell, C. J., et al. (2010). Postprandial metabolism of Pacific bluefin tuna (Thunnus orientalis). J. Exp. Biol. 213, 2379-2385. doi: 10.1242/jeb.043455
JT), Funds from Stanford and the Monterey Bay Aquarium in support of the Tuna Research and Conservation Center (BB), NIH (R01-AG033542 to MB), and DFG grant JA 1884/2-1 (MJ).

\section{ACKNOWLEDGMENTS}

The authors thank Dr. George Somero and his lab for accommodating spectrophotometer use at Hopkins Marine Station, as well as, to the staff of the Tuna Research and Conservation Centre and the Duff Roblin Animal Holding Facility staff (University of Manitoba) for their exceptional assistance with sampling and logistics.

Dickson, K. A., and Graham, J. B. (2004). Evolution and consequences of endothermy in fishes. Physiol. Biochem. Zool. 77, 998-1018. doi: $10.1086 / 423743$

Galli, G. L., Lipnick, M. S., and Block, B. A. (2009). Effect of thermal acclimation on action potentials and sarcolemmal $\mathrm{K}^{+}$channels from Pacific bluefin tuna cardiomyocytes. Am. J. Physiol. 297, R502-R509. doi: 10.1152/ajpregu.90810.2008

Goncalves, R. L., Quinlan, C. L., Perevoshchikova, I. V., Hey-Mogensen, M., and Brand, M. D. (2015). Sites of superoxide and hydrogen peroxide production by muscle mitochondria assessed ex vivo under conditions mimicking rest and exercise. J. Biol. Chem. 290, 209-227. doi: 10.1074/jbc.M114.619072

Graham, J. B., and Dickson, K. A. (2004). Tuna comparative physiology. J. Exp. Biol. 207, 4015-4024. doi: 10.1242/jeb.01267

Heise, K., Puntarulo, S., Pörtner, H. O., and Abele, D. (2003). Production of reactive oxygen species by isolated mitochondria of the Antarctic bivalve Laternula elliptica (King and Broderip) under heat stress. Comp. Biochem. Physiol. C 134, 79-90. doi: 10.1016/S1532-0456(02)00212-0

Jastroch, M., Divakaruni, A. S., Mookerjee, S., Treberg, J. R., and Brand, M. D. (2010). Mitochondrial proton and electron leaks. Essays Biochem. 47, 53-67. doi: 10.1042/bse0470053

Johnston, I. A., Calvo, J., Guderley, H., Fernandez, D., and Palmer, L. (1998). Latitudinal variation in the abundance and oxidative capacities of muscle mitochondria in perciform fishes. J. Exp. Biol. 201, 1-12.

Johnston, I. A., Guderley, H., Franklin, C., Crockford, T., and Kamunde, C. N. (1994). Are mitochondria subject to evolutionary temperature adaptation? J. Exp. Biol. 195, 293-306.

Lambert, A. J., Boysen, H. M., Buckingham, J. A., Yang, T., Podlutsky, A., Austad, S. N., et al. (2007). Low rates of hydrogen peroxide production by isolated heart mitochondria associate with long maximum lifespan in vertebrate homeotherms. Aging Cell 6, 607-618. doi: 10.1111/j.1474-9726.2007.00 312.x

Lauff, R. F., and Wood, C. M. (1997). Effects of training on respiratory gas exchange, nitrogenous waste excretion, and fuel usage during aerobic swimming in juvenile rainbow trout (Oncorhynchus mykiss). Can. J. Fish. Aquat. Sci. 54, 566-571. doi: 10.1139/f96-315

Lauff, R., and Wood, C. (1996). Respiratory gas exchange, nitrogenous waste excretion, and fuel usage during aerobic swimming in juvenile rainbow trout. $J$. Comp. Physiol. B 166, 501-509. doi: 10.1007/BF02338293

Leary, S. C., Lyons, C. N., Rosenberger, A. G., Ballantyne, J. S., Stillman, J., and Moyes, C. D. (2003). Fiber-type differences in muscle mitochondrial profiles. Am. J. Physiol. 285, R817-R826. doi: 10.1152/ajpregu.00058.2003

Marcinek, D. J., Blackwell, S. B., Dewar, H., Freund, E. V., Farwell, C., Dau, D., et al. (2001). Depth and muscle temperature of Pacific bluefin tuna examined with acoustic and pop-up satellite archival tags. Mar. Biol. 138, 869-885. doi: $10.1007 / \mathrm{s} 002270000492$

McClelland, G. B. (2004). Fat to the fire: the regulation of lipid oxidation with exercise and environmental stress. Comp. Biochem. Physiol. B 139, 443-460. doi: 10.1016/j.cbpc.2004.07.003

Moyes, C. D., Buck, L. T., Hochachka, P. W., and Suarez, R. K. (1989). Oxidative properties of carp red and white muscle. J. Exp. Biol. 143, 321-331. 
Moyes, C. D., Mathieucostello, O. A., Brill, R. W., and Hochachka, P. W. (1992). Mitochondrial metabolism of cardiac and skeletal muscles from a fast (Katsuwonus pelamis) and a slow (Cyprinus carpio) fish. Can. J. Zool. 70, 1246-1253. doi: 10.1139/z92-172

Munro, D., Banh, S., Sotiri, E., Tamanna, N., and Treberg, J. R. (2016). The thioredoxin and glutathione-dependent $\mathrm{H}_{2} \mathrm{O}_{2}$ consumption pathways in muscle mitochondria: Involvement in $\mathrm{H}_{2} \mathrm{O}_{2}$ metabolism and consequence to $\mathrm{H}_{2} \mathrm{O}_{2}$ efflux assays. Free Radic. Biol. Med. 96, 334-346. doi: 10.1016/j.freeradbiomed.2016.04.014

Murphy, M. P. (2009). How mitochondria produce reactive oxygen species. Biochem. J. 417, 1-13. doi: 10.1042/BJ20081386

Perevoshchikova, I. V., Quinlan, C. L., Orr, A. L., Gerencser, A. A., and Brand, M. D. (2013). Sites of superoxide and hydrogen peroxide production during fatty acid oxidation in rat skeletal muscle mitochondria. Free Radic. Biol. Med. 61, 298-309. doi: 10.1016/j.freeradbiomed.2013.04.006

Quinlan, C. L., Treberg, J. R., Perevoshchikova, I. V., Orr, A. L., and Brand, M. D. (2012). Native rates of superoxide production from multiple sites in isolated mitochondria measured using endogenous reporters. Free Radic. Biol. Med. 53, 1807-1817. doi: 10.1016/j.freeradbiomed.2012.0 8.015

Santiago, A. P. S., Chaves, E. A., Oliveira, M. F., and Galina, A. (2008). Reactive oxygen species generation is modulated by mitochondrial kinases: correlation with mitochondrial antioxidant peroxidases in rat tissues. Biochimie 90, 1566-1577. doi: 10.1016/j.biochi.2008.06.013

Seifert, E. L., Estey, C., Xuan, J. Y., and Harper, M. E. (2010). Electron transport chain-dependent and -independent mechanisms of mitochondrial $\mathrm{H}_{2} \mathrm{O}_{2}$ emission during long-chain fatty acid oxidation. J. Biol. Chem. 285, 5748-5758. doi: 10.1074/jbc.M109.026203

St Pierre, J., Buckingham, J. A., Roebuck, S. J., and Brand, M. D. (2002). Topology of superoxide production from different sites in the mitochondrial electron transport chain. J. Biol. Chem. 277, 44784-44790. doi: 10.1074/jbc.M2072 17200
Starkov, A. A., and Fiskum, G. (2003). Regulation of brain mitochondrial $\mathrm{H}_{2} \mathrm{O}_{2}$ production by membrane potential and $\mathrm{NAD}(\mathrm{P}) \mathrm{H}$ redox state. J. Neurochem. 86, 1101-1107. doi: 10.1046/j.1471-4159.2003.01908.x

Starkov, A. A., Fiskum, G., Chinopoulos, C., Lorenzo, B. J., Browne, S. E., Patel, M. S., et al. (2004). Mitochondrial $\alpha$-ketoglutarate dehydrogenase complex generates reactive oxygen species. J. Neurosci. 24, 7779-7788. doi: 10.1523/JNEUROSCI.1899-04.2004

Stevens, E. D., Kanwisher, J. W., and Carey, F. G. (2000). Muscle temperature in free-swimming giant Atlantic bluefin tuna (Thunnus thynnus L.). J. Therm. Biol. 25, 419-423. doi: 10.1016/S0306-4565(00)00004-8

Weber, J. M., and Haman, F. (1996). Pathways for metabolic fuels and oxygen in high performance fish. Comp. Biochem. Physiol. A 113, 33-38 doi: 10.1016/0300-9629(95)02063-2

Wegner, N. C., Snodgrass, O. E., Dewar, H., and Hyde, J. R. (2015). Whole-body endothermy in a mesopelagic fish, the opah, Lampris guttatus. Science 348, 786-789. doi: 10.1126/science.aaa8902

Whitlock, R. E., Hazen, E. L., Walli, A., Farwell, C., Bograd, S. J., Foley, D. G., et al. (2015). Direct quantification of energy intake in an apex marine predator suggests physiology is a key driver of migrations. Sci. Adv. 1:e1400270. doi: $10.1126 /$ sciadv. 1400270

Conflict of Interest Statement: The authors declare that the research was conducted in the absence of any commercial or financial relationships that could be construed as a potential conflict of interest.

Copyright (C) 2017 Wiens, Banh, Sotiri, Jastroch, Block, Brand and Treberg. This is an open-access article distributed under the terms of the Creative Commons Attribution License (CC BY). The use, distribution or reproduction in other forums is permitted, provided the original author(s) or licensor are credited and that the original publication in this journal is cited, in accordance with accepted academic practice. No use, distribution or reproduction is permitted which does not comply with these terms. 